\title{
High-gain power recycling of a Fabry-Perot Michelson interferometer for a gravitational-wave antenna
}

\author{
Shuichi Sato, Masatake Ohashi, Masa-Katsu Fujimoto, Mitsuhiro Fukushima, \\ Koichi Waseda, Shinji Miyoki, Nergis Mavalvala, and Hiroaki Yamamoto
}

\begin{abstract}
Power recycling was implemented on a fully suspended prototype interferometer with arm lengths of $20 \mathrm{~m}$. A wave-front-sensing technique for alignment control of the suspended mirrors was also implemented, which allowed for several hours of stable operation. A power-recycling gain of greater than 12 was achieved, a significant increase over the highest gain in a suspended mirror Fabry-Perot Michelson interferometer reported to date. (C) 2000 Optical Society of America

OCIS codes: $120.3180,000.2780,120.2230,350.1270$.
\end{abstract}

\section{Introduction}

One of the most exciting topics in both physics and astronomy in the coming century may be direct detection of gravitational waves. In addition to the interest in the nature of gravity as a proof of general relativity, gravitational-wave detectors have the potential to open a new window into the universe. Because of the overwhelming transmissivity of gravitational waves - the universe is more transparent to them than even to neutrinos-the gravitational wave is expected to carry much more information about distant astrophysical phenomena. For the same reason, namely, that gravitational waves interact weakly with matter, the gravitationalwave-induced strain from some of the most promising

When this research was performed, S. Sato (sato@icrr.u-tokyo. ac.jp), M. Ohashi, M.-K. Fujimoto, M. Fukushima, and K. Waseda were with the National Astronomical Observatory, 2-21-1, Osawa, Mitaka, Tokyo 181-8588, Japan. S. Miyoki was with the Institute for Cosmic Ray Research, University of Tokyo, 5-1-5, Kashiwanoha, Kashiwa, Chiba 277-8582, Japan. N. Mavalvala and H. Yamamoto were with the Laser Interferometer GravitationalWave Observatory Laboratory, California Institute of Technology, MS 18-34, Pasadena, California 91125. S. Sato is now with the Kamioka Observatory, Institute for Cosmic Ray Research, University of Tokyo, Higashi-Mozumi, Kamioka, Yoshiki, Gifu 506-1205, Japan. M. Ohashi is with the Institute for Cosmic Ray Research, The University of Tokyo, 5-1-5, Kashiwanoha, Kashiwa, Chiba 277-8582, Japan.

Received 8 February 2000; revised manuscript received 23 May 2000.

0003-6935/00/254616-05\$15.00/0

(C) 2000 Optical Society of America astrophysical sources is expected to be smaller than $10^{-21}$.1

To detect gravitational waves from astrophysical sources, several ground-based large-scale [the Laser Interferometer Gravitational-Wave Observatory $(\mathrm{LIGO})^{2}$ and the Italian-French Laser Interferometer Collaboration (VIRGO) ${ }^{3}$ ] and medium-scale [GEO600 (Ref. 4) and TAMA300 (Ref. 5)] laser interferometric detectors are now under construction. (GEO, German-British Cooperation for GravityWave Experiment; TAMA, Japanese Interferometric Gravitational-Wave Detector Project). All these detectors employ variants of a Michelson interferometer, with novel additions to the optical configuration to increase their sensitivity to gravitational waves. ${ }^{6}$

Typically, the sensitivity of the interferometer is expected to be limited by seismic noise at low frequencies (below $10-40 \mathrm{~Hz}$ ), by thermally driven fluctuations of the suspended mirrors at intermediate frequencies (below $100 \mathrm{~Hz}$ ), and by shot noise at higher frequencies. The shot noise, which is due to photon-counting statistics, is proportional to the square root of the light power, whereas the signal strength scales with laser power. The signal-tonoise ratio of the interferometer is then proportional to the inverse of $\sqrt{P}$, and the shot-noise-limited sensitivity can be improved by means of increasing the light power incident to the interferometer. One technique to increase the effective power in the gravitational-wave detector is power recycling. ${ }^{7}$ A schematic representation of the optical configuration for a power-recycled gravitational-wave interferometer is shown in Fig. 1. Resonant Fabry-Perot cavities in each arm of the Michelson interferometer enhance the phase sensitivity of the detector. Since 


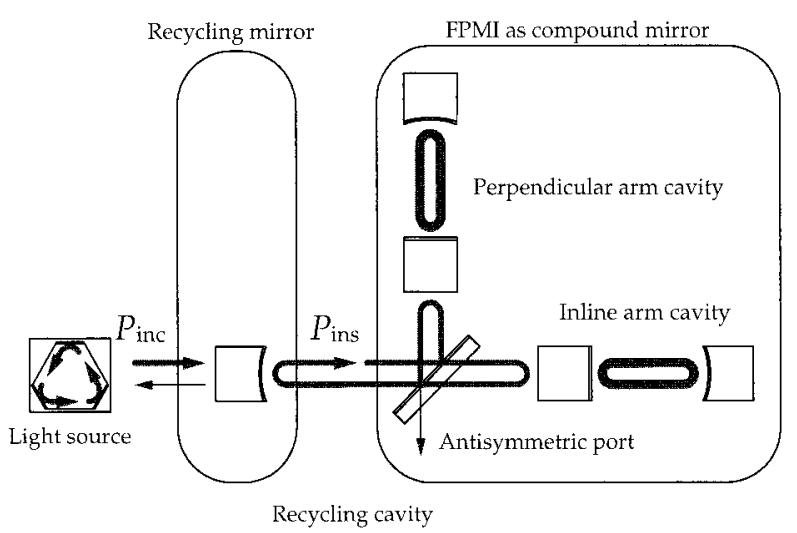

Fig. 1. Optical design of power recycling. The recycling mirror is placed between the light source and the FPMI as a compund mirror. These two mirrors make up the recycling cavity.

the antisymmetric port of the Michelson interferometer is operated on a dark fringe, most of the incident light returns toward the laser. A partially transmitting mirror placed between the beam splitter and the laser recycles this light back into the interferometer, increasing the effective power and thereby improving the shot-noise-limited sensitivity by a factor $\sqrt{G_{\text {rec}}}$, where $G_{\text {rec }}$ is the power-recycling gain.

Our main purpose in this study is to apply the power-recycling technique to a suspended Michelson interferometer with Fabry-Perot arm cavities for full testing of its feasibility for gravitational-wave detectors with this optical configuration. Power recycling was implemented in the TAMA project's 20 -m prototype interferometer, which can most closely simulate a full-scale antenna, and we demonstrated a powerrecycling gain of the order of 10 , which is required for TAMA300 and also for other gravitational-wave detectors.

\section{Power Recycling}

Referring to the optical arrangement in Fig. 1, we see that the power-recycling mirror is placed between the light source and the rest of the interferometer. Since the fringe at the antisymmetric output of the recombined Fabry-Perot Michelson interferometer (FPMI) is controlled so as to keep the antisymmetric port dark, the FPMI can be regarded as a compound mirror with variable (complex) reflectivity. The power-recycling cavity is composed of the powerrecycling mirror and this compound mirror. Power is built up in the recycling cavity by means of maintaining the appropriate resonance conditions in the FPMI and the recycling cavity. The effective power incident on the beam splitter (main interferometer) is then increased by this optical power enhancement. The power-recycling gain (power enhancement factor) is defined as

$$
G=\frac{P_{\mathrm{ins}}}{P_{\mathrm{inc}}}=\left(\frac{t_{\mathrm{RM}}}{1-r_{\mathrm{RM}} r_{\mathrm{FPMI}}}\right)^{2},
$$

where, $r_{\mathrm{RM}}$ and $r_{\mathrm{FPMI}}$ are the amplitude reflectivities of the recycling mirror and of the FPMI, respectively. In general, losses of a few parts per million (ppm) per encounter with a mirror surface can result in losses of the order of a percent in the FPMI, depending on the finesse of Fabry-Perot cavities and on the interference conditions set up in the Michelson interferometer. Assuming negligibly small loss for the powerrecycling mirror, $A_{\mathrm{RM}}$ compared with total loss in the FPMI, $A_{\text {FPMI }}, G$ is approximated as

$$
G \simeq A_{\mathrm{FPMI}}^{-1}
$$

From this expression we see that the maximum attainable recycling gain is limited by the total losses in the interferometer. Consequently, minimizing losses in the interferometer is critical for realizing a high power-recycling gain. High-gain recycling is essentially equivalent to building-up a high-quality, lowloss interferometer.

\section{Experimental Setup}

The experimental setup and schematic of the control system are shown in Fig. 2. To mitigate the effects of acoustic noise and of fluctuations of the refractive index of the air, all the mirrors that make up the arm cavities, the power-recycling cavity, the mode cleaner (MC) cavity, and the beam splitter are housed in a vacuum system maintained at $10^{-6}$ Torr. The interferometric optical components are suspended as masses at the end of a two-stage pendulum, where the eigenmodes of the pendulum are magnetically damped with eddy currents in the intermediate masses. The mirror suspensions are placed on optical breadboards, which are the uppermost masses of spring-mass stacks to provide further isolation from seismic fluctuations. A coil-magnet actuation system is used for microscopic control of the positions and angles of each suspended optic.

There are four longitudinal degrees of freedom to be controlled for the power-recycled FPMI. Error signals to sense deviations from the resonance of each optical length are obtained by a frontal modulation scheme, ${ }^{8}$ which uses a combination of the PoundDrever-Hall technique ${ }^{9}$ and the Schnupp asymmetry readout scheme. The light source is a diode-pumped Nd:YAG laser (MISER Model 122-1064-500-F, Lightwave Electronics Corp.) with an emission wavelength of $1064 \mathrm{~nm}$ and an output power of $511 \mathrm{~mW}$. The laser light is phase modulated at $40 \mathrm{MHz}$ for the interferometric controls and at $18 \mathrm{MHz}$ for locking the MC cavity.

The 20-m prototype interferometer is equipped with a suspended Fabry-Perot precavity as a MC, whose main function is to reduce spatial higher-order modes of Gaussian beam and beam-pointing fluctuations of the laser light. Since the electro-optic modulators used to impose the rf sidebands on the light can degrade the spatial mode, it is advantageous to place the electro-optic modulators before the MC and to transmit the $40-\mathrm{MHz}$ rf phase-modulation sidebands resonantly for interferometric control through 


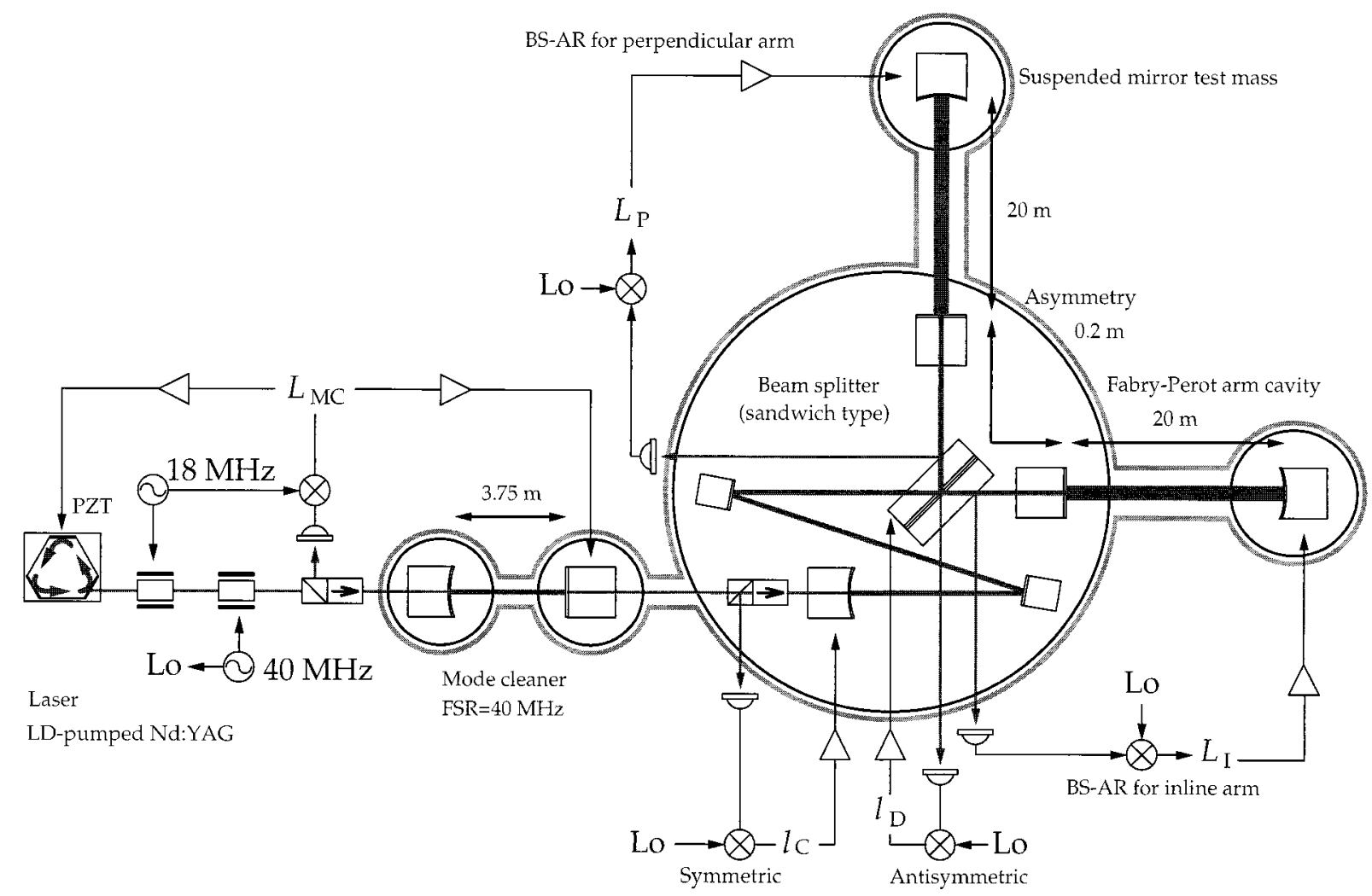

Fig. 2. Experimental setup of 20-m interferometer for power recycling. BS-AR, reflection from beam splitter antireflection coating; PZT, piezoelectric transducer; LD, laser diode; FSR, free spectral range.

the MC cavity by means of choosing its length to be $3750 \mathrm{~mm}$ to make its free spectral range match 40 $\mathrm{MHz}$.

Another important function of the MC cavity is that it serves as a stable frequency reference for the laser. We obtained the error signal for maintaining the $\mathrm{MC}$ cavity on resonance by imposing $18-\mathrm{MHz}$ phase-modulation sidebands on the incident light and demodulating the light reflected from the MC cavity with an $18-\mathrm{MHz}$ local oscillator. The appropriately filtered error signal was then split into two feedback paths: At low frequencies $(<30 \mathrm{~Hz})$ the MC mirrors are actuated to stabilize its length, and at higher frequencies $(>30 \mathrm{~Hz})$ the laser frequency is slaved to the MC cavity by a control signal applied to the piezoelectric transducer actuator of the laser. In other words, the emission frequency of laser light was locked to the length of the MC cavity, and the frequency was stabilized by use of the MC cavity as a frequency reference. The frequency noise of the laser was thus stabilized down to $10^{-3} \mathrm{~Hz} / \sqrt{\mathrm{Hz}}$ at 1 $\mathrm{kHz}$, which facilitated acquisition of a lock of the main interferometer with relatively low-bandwidth feedback loops.

A small fraction of the light reflected from the resonant arm cavities, picked off as a reflection from the antireflection-coated side of the beam splitter, was used to control the in-line and perpendicular arm cavity lengths, $L_{I}$ and $L_{P}$, respectively. Each of these pick-off fields contains an admixture of signals proportional to both $L_{I}$ and $L_{P}$, because the optical fields are circulating and mixing inside the powerrecycling cavity. The two loops, however, can lock both arm cavities independently and have enough gain margin to maintain resonance in the arm cavities even if the power-recycling cavity and the Michelson loops are unlocked. This control scheme enabled simple and fast acquisition of the interferometer lock. The signal for the average powerrecycling cavity length $l_{C}$ was sensed at the symmetric output with feedback to the recycling mirror actuator and while Michelson differential degree of freedom $l_{D}$ was sensed at the antisymmetric port of the beam splitter. All signals were sensed with photodetectors tuned at $40 \mathrm{MHz}$ followed by demodulation with a suitable phase, filtered and fed back to the coil-magnet actuators.

In addition to the longitudinal degrees of freedom, each of the six optics has two angular degrees of freedom (horizontal and vertical tilts). Among the twelve angular degrees of freedom, differential-mode misalignment of the arm cavity mirrors can significantly degrade the power-recycling gain that is due imperfect interference at the beam splitter, which results in a lower reflectivity, $r_{\mathrm{FPMI}}$, and, therefore, lower recycling gain. An alignment control scheme based on a wave-front-sensing technique was applied to all angular degrees of freedom of a fixed-mirror tabletop-scale power-recycled FPMI interferometer. ${ }^{10}$ We use the same technique but stabilize only 


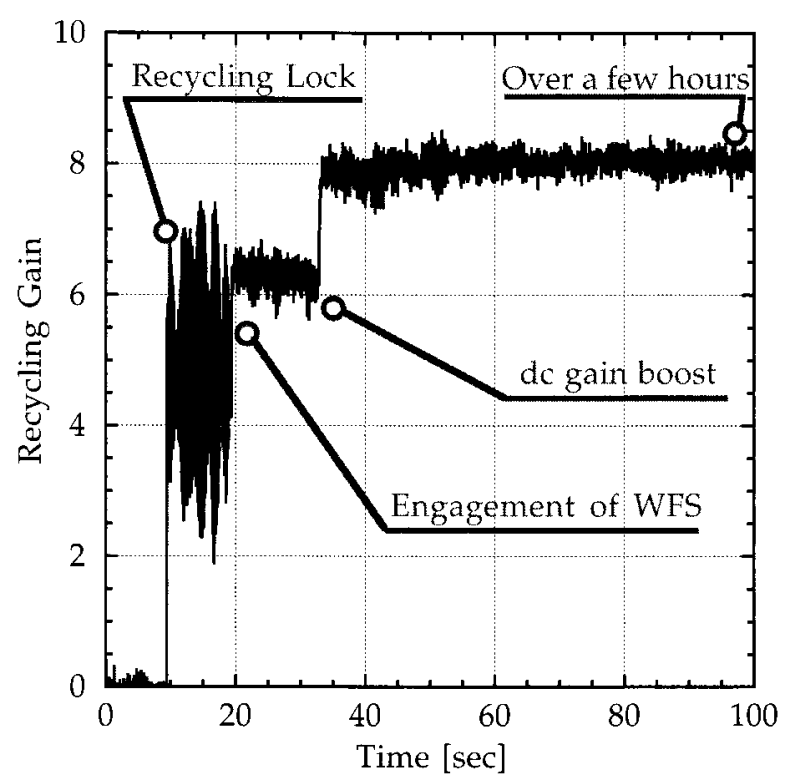

Fig. 3. Recycling gain with $r_{\mathrm{RM}}^{2}=0.7$. Without alignment control the recycling gain was fluctuating near 5 . It went up to $\sim 8$, and the fluctuations were stabilized when the wave-front sensing servo was engaged.

the most problematic angular fluctuations. Some of the light at the antisymmetric port was diverted to a segmented 40-MHz-tuned photodetector, and the demodulated signal was filtered and fed back into the rear mirrors of both arm cavities differentially.

The typical sequence for bringing the full interferometer into longitudinal lock was as follows: Upon engaging the four longitudinal control loops the two arm cavities locked first-within a few seconds in most cases - then the whole interferometer acquired lock. Without interferometric alignment control, this operating state could be maintained for more than $1 \mathrm{~h}$ in the absence of impulsive seismic excitations.

The power-recycling gain during a 100-s lock stretch is shown in Fig. 3. Since only carrier light resonates in the arm cavity, this enhancement factor of transmitted light corresponds to the carrier recycling gain, $G_{0}$. The large fluctuations $(>50 \%)$ seen during the first $20 \mathrm{~s}$ were caused primarily by a vertical tilt motion of suspended mirrors with an $8-\mathrm{Hz}$ eigenfrequency. The fluctuations in power-recycling gain were suppressed by engagement of the alignment servo, and finally the power-recycling gain was increased with dc gain boost, which brought the interferometer into an improved dc alignment state.

The finesse of each arm cavity was designed to be 130 to simulate the design of the full-scale detector. With a power-recycling mirror reflectivity, $r_{\mathrm{RM}}^{2}=0.7$, and assuming the reflection efficiency of each cavity to be $99 \%$, the observed recycling gain of 8 is almost the maximum that can be expected. In an alternative setup, using a recycling mirror with higher reflectivity, $r_{\mathrm{RM}}^{2}=0.9$, the power-recycling gain was

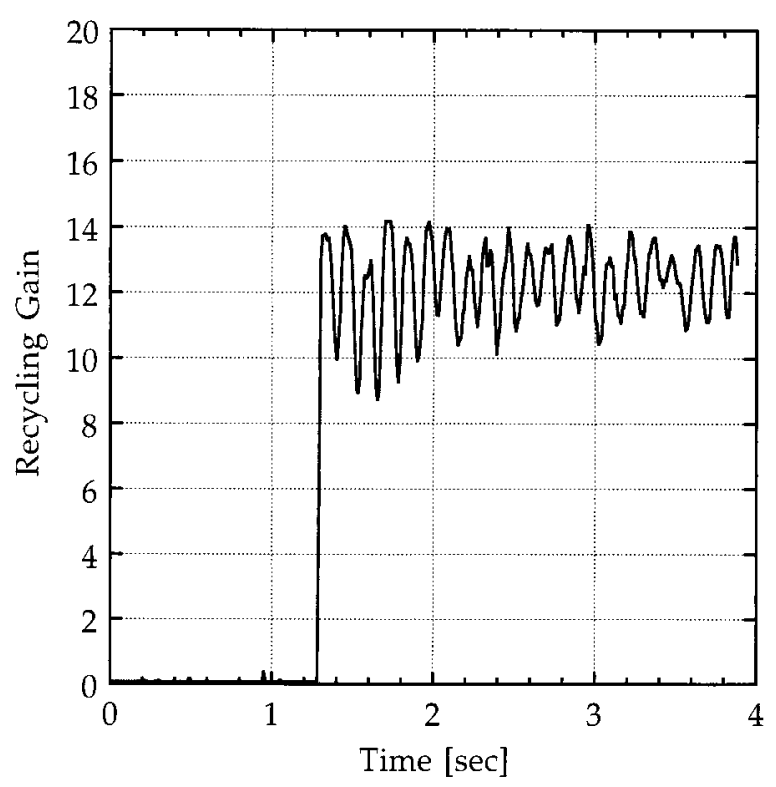

Fig. 4. Recycling gain with $r_{\mathrm{RM}}^{2}=0.9$. As optical matching of the recycling mirror and the compound FPMI mirror was improved, close to the maximum possible gain (for these optical parameters) was observed. The wave-front sensor servo was not engaged.

increased to $G_{0}>12$, as shown in Fig. 4. In this configuration the optical matching of the recycling cavity with respect to the reflectivity of the FPMI was improved to near-critical matching, but it was still slightly overcoupled.

Two features of power recycling were found to be particularly important. First, to achieve a high recycling gain, careful control of mirror losses was indispensable. We have confirmed that it is possible to fabricate ultra-low-loss optics with a total loss of less than $30 \mathrm{ppm}$ per optic, ${ }^{11}$ which is low enough to achieve a power-recycling gain of 10 in the full-scale detector. To our knowledge, this was the first measurement of total loss for 10-mm beam and the best value for mirrors used in prototype-class interferometers. Second, implementation of wave-frontsensing alignment control was quite important for maintaining high reflection efficiency for both arm cavities and for reducing contrast defect. The recycling gain was dramatically stabilized and enhanced by use of the wave-front sensor. Stable operation of the power-recycled FPMI was realized to maintain lock for a few hours.

\section{Summary}

In conclusion, the experiment described was a demonstration of the highest power-recycling gain for a suspended mirror Fabry-Perot Michelson interferometer (FPMI) reported to date. With continuous operation of the TAMA $300-\mathrm{m}$ antenna imminent, the 20-m prototype experiments have provided a timely demonstration of power-recycling in a fully suspended FPMI with optical parameters similar to those of the full-scale detector. This result will also 
form the basis of the optical design of a full-scale detector project such as the Large-Scale Cryogenic Gravitational-Wave Telescope. ${ }^{12}$

The authors thank K. Ito and co-workers at Japan Aviation Electronics Co, Ltd., for their technical support. This study was partially supported by the Japan Society for the Promotion of Science and a grant-in-aid for Creative Basic Research of the Ministry of Education (09NP0801). N. Mavalvala and H. Yamamoto were supported under National Science Foundation (NSF) Cooperative Agreement PHY9210038 .

\section{References}

1. K. S. Thorne, "Gravitational radiation," in 300 Years of Gravitation (Cambridge University, Cambridge, UK, 1987), p. 330.

2. A. Abramovici, W. E. Althouse, R. W. P. Drever, Y. Gursel, S. Kawamura, F. J. Raab, D. Shoemaker, L. Sievers, R. E. Spero, K. S. Thorne, R. E. Vogt, R. Weiss, S. E. Whitcomb, and M. E. Zucker, "LIGO: the Laser Interferometer GravitationalWave Observatory," Science 256, 325-333 (1992).

3. C. Bradaschia, R. Del Fabbro, A. Di Virgilio, A. Giazotto, H. Kautzkey, V. Montelatici, D. Passuello, A. Brillet, O. Cregut, P. Hello, C. N. Man, P. T. Manh, A. Marraud, D. Shoemaker, J.-Y. Vinet, F. Barone, L. Di Fiore, L. Milano, G. Russo, J. M. Aguirregabiria, H. Bel, J.-P. Duruisseau, G. Le Denmat, P. Tourrenc, M. Capozzi, M. Longo, M. Lops, I. Pinto, G. Rotoli, T. Damour, S. Bonazzola, J. A. Marck, Y. Gourghoulon, L. E. Holloway, F. Fuligni, V. Iafolla, and G. Natale, "The VIRGO project: a wide band antenna for gravitational wave detection,” Nucl. Instrum. Methods Phys. Res. A 289, 518-525 (1990).

4. K. Danzmann, H. Luck, A. Rudiger, R. Schilling, M. Schrempel, W. Winkler, J. Hough, G. P. Newton, N. A. Robertson, A. M. Campbell, J. E. Logan, D. I. Robertson, K. A. Strain, J. R. J. Bennet, V. Kose, M. Kuhne, B. F. Schutz, D. Nicolson, J. Shuttleworth, H. Welling, P. Aufmuth, R. Rinkleff, A. Tun- nermann, and B. Willke, "Proposal for a $600 \mathrm{~m}$ laserinterferometric gravitational wave antenna," Internal Rep. MPQ 190 (Max-Planck-Institut für Quantenoptik, Garching, Germany, 1994).

5. K. Tsubono, "300m laser interferometer gravitational wave detector (TAMA300) in Japan," in Proceedings of the First Edoardo Amaldi Conference on Gravitational Wave Experiments, Frascati, 1994 (World Scientific, Singapore, 1995), pp. 112-114.

6. P. R. Saulson, Fundamentals of Interferometric Gravitational Wave Detectors (World Scientific, Singapore, 1994).

7. R. W. P. Drever, "Interferometric detectors for gravitational radiation," in Gravitational Radiation (North-Holland, Amsterdam, 1983), pp. 321-338.

8. M. W. Regehr, F. J. Raab, and S. E. Whitcomb, "Demonstration of a power-recycled Michelson interferometer with FabryPerot arms by frontal modulation," Opt. Lett. 20, 1507-1509 (1995).

9. R. W. P. Drever, J. L. Hall, F. V. Kowalski, J. Hough, G. M. Ford, A. J. Munleyand, and H. Ward, "Laser phase and frequency stabilization using an optical resonator," Appl. Phys. B 31, 97-105 (1983).

10. N. Mavalvala, D. Sigg, and D. Shoemaker, "Experimental test of an alignment-sensing scheme for a gravitational-wave interferometer," Appl. Opt. 37, 7743-7746 (1998).

11. S. Sato, S. Miyoki, M. Ohashi, M.-K. Fujimoto, T. Yamazaki, M. Fukushima, A. Ueda, K. Ueda, K. Watanabe, K. Nakamura, K. Etoh, N. Kitajima, K. Ito, and I. Kataoka, "Loss factors of mirrors for a gravitational wave antenna," Appl. Opt. 38, 2880-2885 (1999).

12. K. Kuroda, M. Ohashi, S. Miyoki, D. Tatsumi, S. Sato, H. Ishizuka, M.-K. Fujimoto, S. Kawamura, R. Takahashi, T. Yamazaki, K. Arai, M. Fukushima, K. Waseda, S. Telada, A. Ueda, T. Shintomi, A. Yamamoto, T. Suzuki, Y. Saito, T. Haruyama, N. Sato, K. Tsubono, K. Kawabe, M. Ando, K. Ueda, H. Yoneda, M. Musha, N. Mio, S. Moriwaki, A. Araya, N. Kanda, and M. E. Tobar, "Large-Scale Cryogenic Gravitational Wave Telescope,” Int. J. Mod. Phys. D 8, 557-579 (1999). 